\title{
Inferred Genetic Architecture Underlying Evolution in a Fossil Stickleback Lineage
}

\author{
Yoel E. Stuart \\ Loyola University Chicago, ystuart@luc.edu \\ Matthew P. Travis \\ Rowan University \\ Michael A. Bell \\ University of California Museum of Paleontology
}

Follow this and additional works at: https://ecommons.luc.edu/biology_facpubs

Part of the Biology Commons

\section{Author Manuscript}

This is a pre-publication author manuscript of the final, published article.

\section{Recommended Citation}

Stuart, Yoel E.; Travis, Matthew P.; and Bell, Michael A.. Inferred Genetic Architecture Underlying Evolution in a Fossil Stickleback Lineage. Nature Ecology and Evolution, 4, : pages1549-1557, 2020. Retrieved from Loyola eCommons, Biology: Faculty Publications and Other Works, http://dx.doi.org/10.1038/ s41559-020-01287-x

This Article is brought to you for free and open access by the Faculty Publications and Other Works by Department at Loyola eCommons. It has been accepted for inclusion in Biology: Faculty Publications and Other Works by an authorized administrator of Loyola eCommons. For more information, please contact ecommons@luc.edu.

(c) Springer Nature Limited, 2020. 
1 Title: Inferred genetic architecture underlying evolution in a fossil stickleback lineage

3 Authors: Yoel E. Stuart ${ }^{1, *}$, Matthew P. Travis ${ }^{2}$, and Michael A. Bell ${ }^{3}$

$4{ }^{1}$ Department of Biology, Loyola University Chicago, Chicago, IL, USA

$5 \quad{ }^{2}$ Department of Biological Sciences, Rowan University, Glassboro, NJ, USA

$6 \quad{ }^{3}$ University of California Museum of Paleontology, Berkeley, CA, USA

7 * Corresponding author: ystuart@1uc.edu ORCID ID: 0000-0003-1235-6277

9 Inferring the genetic architecture of evolution in the fossil record is difficult because genetic

10 crosses are impossible, the acquisition of DNA is usually impossible, and phenotype-genotype

11 maps are rarely obvious. However, such inference is valuable because it reveals the genetic basis

12 of microevolutionary change across many more generations than is possible in studies of extant

13 taxa, thereby integrating microevolutionary process and macroevolutionary pattern. Here, we

14 infer the genetic basis of pelvic skeleton reduction in Gasterosteus doryssus, a Miocene

15 stickleback fish from a finely resolved stratigraphic sequence that spans nearly 17,000 years.

16 Reduction in pelvic score, a categorical measure of pelvic structure, resulted primarily from

17 reciprocal frequency changes of two discrete phenotypic classes. Pelvic vestiges also showed

18 left-side-larger asymmetry. These patterns implicate Pitx 1, a large-effect gene whose deletion

19 generates left-larger asymmetry of pelvic vestiges in extant, closely-related Gasterosteus

20 aculeatus. In contrast, reductions in lengths of the pelvic girdle and pelvic spines resulted from

21 directional shifts of unimodal, continuous trait distributions, suggesting an additional suite of

22 genes with minor, additive pelvic effects, again like G. aculeatus. Similar genetic architectures

23 explain shared but phyletically independent patterns across 10 million years of stickleback

24 evolution.

26 We studied the last $\sim 16,500$ years of a $\sim 108,275$ year-long fossil $G$. doryssus sequence ${ }^{1,2}$. The

27 entire sequence contains two lineages of G. doryssus. Lineage I existed during the first 92,012

28 years of the sequence and had a vestigial pelvic girdle and fewer than three dorsal spines, on

29 average. At 92,012 years, lineage I was replaced within 125 years by lineage II (Extended Data

30 2), whose source was a parapatric G. doryssus population from outside the depositional basin ${ }^{3}$.

31 At the replacement event, lineage II invariably had a robust pelvis and three dorsal spines ${ }^{2}$. 
32 Lineage II subsequently evolved vestigial armor phenotypes, similar to those of lineage $\mathrm{I}^{1}$, under 33 directional natural selection ${ }^{4}$ over 16,750 years ${ }^{3}$. Armor reduction in Lineage II included

34 reduction in the size and complexity of the pelvic girdle and pelvic spines.

Such pelvic reduction exists in many extant lake populations of . aculeatus $^{5}$ and is

36 likely also driven by natural selection ${ }^{6,7}$. The major gene underlying pelvic reduction in $G$.

37 aculeatus is usually Pitxl (Pituitary homeobox 1$)^{7-12}$ (but see ${ }^{13}$ ). Loss occurs through deletion

38 mutations in the Pel enhancer region that reduce pelvis-specific Pitxl expression ${ }^{7,12}$. Deletion

39 mutations of $\mathrm{Pel}$ act recessively and their phenotypic effects on pelvic score (PS: a categorical

40 metric of pelvis size and complexity; Methods) segregate in multimodal, near Mendelian fashion

41 in G. aculeatus $^{9,10}$. Moreover, because a paralogous gene, Pitx2, presumably also contributes to

42 pelvic girdle formation but is expressed more on the left side than the right ${ }^{14}$, reduced Pitxl

43 expression results in a directionally asymmetrical, left-larger pelvic vestige in $G$.

44 aculeatus $^{5,9,11,13,15}$ (as well as other vertebrates ${ }^{9,14,16}$ ). Because Pitxl has repeatedly played a

45 major role in pelvic reduction in G. aculeatus, and because G. aculeatus is closely related to $G$.

46 doryssus $^{17}$, Pitxl is a good candidate gene for pelvic reduction in G. doryssus. To infer whether

47 Pitxl is responsible for major pelvic reduction in G. doryssus ${ }^{1,3}$, we examined our fossil data for

48 a Mendelian pattern of pelvic scores and for left-larger pelvic vestige asymmetry (Question 1).

Pelvic reduction in extant G. aculeatus also mapped to several genomic regions with minor, additive effects ${ }^{9-11,18}$. To infer whether genes with small effects also contributed to pelvic reduction in G. doryssus (Question 2), we used the fact that pelvic score in G. doryssus did not

52 decline immediately after the fully armored lineage II appeared in the temporal sequence ${ }^{3}$.

53 Although reduction of other, non-pelvic armor traits (i.e., numbers of dorsal spines and touching 54 predorsal pterygiophores) began to evolve immediately ${ }^{3}$, pelvic score remained static in lineage 55 II for $\sim 3,500$ years before declining. We examined new data for pelvic girdle length and pelvic spine length to ask whether those traits had also experienced delayed reduction. If yes, this

57 suggests that natural selection for armor loss did not initially include selection for reduced 58 pelvises. If not, however, and reduction began immediately, this suggests that pelvic reduction in G. doryssus was polygenic and that there was variation in minor genes that allowed some pelvic 60 reduction in response to natural selection before the appropriate, hypothesized mutations in Pitx 1 61 arose and became the basis for more extensive pelvic reduction. 


\section{Results}

64 Question 1. Is Pitx1 responsible for the reduction of pelvic score observed in lineage II of $G$. doryssus, $\sim 3500$ years after lineage II appeared? We measured the lengths of the right and left

66 pelvic vestiges from 815 specimens from temporal sequence L (Methods, Extended Data 2). A 67 paired t-test indicated that pelvic vestiges were significantly larger on the left side (mean rightminus-left difference $\left.=-0.29 \mathrm{~mm}, \mathrm{t}_{814}=-8.39, \mathrm{p}<0.0001\right)$. The left vestige was larger in $73.5 \%$ of pelvic-reduced specimens, significantly more than half $\left(\chi_{1}^{2}=95.31, \mathrm{p}<0.0001\right.$; Figure 1$)$, corroborating a preliminary finding using a much smaller sample size ${ }^{13}$. This left-bias was not strongly influenced by the extent of pelvic reduction (i.e., pelvic score category; Methods; $\chi_{9}^{2}=$ 13.05, $\mathrm{p}=0.16$, Table S1).

The distributions of pelvic scores (PS) of lineage II specimens were multimodal during reduction. In temporal sequence $\mathrm{L}$, all but three of the 595 specimens in the first 12 samples following replacement of lineage I by lineage II had a full pelvis (PS 3.0; Figure 2), spanning the first 2,750 years of lineage II (Table S2). Then, mean PS declined to PS 2.92, where it remained static for another 750 years (Figure 2, Table S2). This decline to 2.92 was caused by appearance of only two specimens with extreme pelvic reduction (PS $\leq 1)$ out of 66 (Table S2). After another 500 years, a third mode formed at PS 2.0, driven mostly by an increase of fish with vestigial pelvises of PS 1.0 (Figure 2, Table S2). PS declined after that to a mean value of about 1.0 by 10,000 years after replacement (Figure 2, Table S2). This new phenotype is indistinguishable from pelvic vestiges that had characterized lineage I for 92,012 years before lineage II appeared ${ }^{1,3}$. Specimens that lack the pelvis entirely appear near the middle of the sequence but never become very frequent (maximum PS $0=16.7 \%, 13,750$ years after replacement; Table S2). We are unsure why PS 3.0 individuals do not disappear completely, though low frequency dispersal from the lineage II source population could explain this pattern; occasional full-pelvis migrants were also detected in the first 92,012 years of the fossil sequence ${ }^{1}$ (Extended Data 2; Methods).

Our evidence thus suggests that Pitxl was indeed the major gene responsible for pelvic

912 , Figure 4) resulted largely from changes in the relative frequencies of specimens in two

92 discrete, contrasting phenotypic classes (i.e., PS 1.0 and 3.0; Figure 3, Table S2) rather than

93 from a gradual change from PS 3.0 toward PS 1.0 in the position of a single mode. This is 
94 consistent with the action of an allele of large effect, like deletion of a pelvic enhancer of 95 Pitx $I^{7,9,15}$. Second, left-larger directional asymmetry of pelvic vestiges (Figure 1) implicates 96 Pitxl as the primary factor for pelvic reduction in lineage II of G. doryssus because (i) only six

97 genes in the vertebrate genome are known to be related to directional asymmetry in limb bud 98 tissue during development ${ }^{14}$; (ii) left-biased asymmetry is a known outcome of deletion 99 mutations that reduce expression of Pitxl in vertebrates ${ }^{15}$; and (iii) in extant G. aculeatus, 100 vestigial pelvic phenotypes that map to the Pitxl locus tend to be larger on the left side? 101 Third, the lag time of 3500 years observed before reduction of pelvic score in G. doryssus 102 lineage II (Figure 2, Figure 4) can be explained by a recessive mutation like Pitx l, as follows.

103 Natural selection favored armor reduction in lineage II immediately following its replacement of 104 lineage $\mathrm{I}^{3,4} 5,6,1920,21$. This is evident from immediate reduction in the mean number of dorsal 105 spines (armor structures ${ }^{22}$ ), the mean number of touching pre-dorsal pterygiophores ${ }^{3}$ (which are 106 structurally and likely functionally related to the dorsal spines), as well as in mean pelvic girdle 107 length and mean pelvic spine length (this study; see Question 2, Figure 4). Despite selection for 108 armor reduction ${ }^{4}$, however, pelvic score remained at 3.0 for several thousand years (Table S2).

109 This suggests that the founders of lineage II initially lacked a Pitxl allele for pelvic reduction, or 110 carried it at such low frequencies that individuals that expressed the reduced allele (ie., 111 homozygotes) were too rare for directional selection to act efficiently. This could make sense 112 because the parapatric source population for lineage II could have been under purifying selection 113 to remove pelvic-reducing deletion mutations of large effect in $P i t x I^{2}$; this source population 114 coexisted with predatory fishes that were present elsewhere in the larger drainage (but not in the 115 depositional environment sampled here) $)^{2,23,24}$. Fish predators select for armor ${ }^{5,6,19-21}$.

116 Eventually, however, reduction of pelvic score proceeded in Lineage II based on alleles

117 that reduce Pitxl expression during development of the pelvis. Where would low-armor Pitx 1

118 variants have come from? In extant G. aculeatus, de novo deletion mutations of the PelA

119 enhancer occur at a remarkably high rate ${ }^{12}$. PelA lies within a stretch of fragile DNA that 120 experiences deletion mutations nearly four orders of magnitude faster than in other parts of the 121 threespine stickleback genome and than is typical of vertebrate genomes ${ }^{12}$, increasing the 122 likelihood that enhancer mutations will be generated. Several G. aculeatus populations have 123 evolved pelvic loss over the last 15,000 years by independently acquiring deletion mutations in 124 the PelA enhancer region of Pitx ${ }^{7}$. This suggests that appropriate Pitxl mutations in G. doryssus 
125 lineage I could have arisen often in the environment sampled here. However, if they were also 126 recessive (as in modern stickleback ${ }^{9,10}$ ), these mutations would have had to drift to an

127 appreciable frequency before homozygotes would occur and selection could drive the deletion

128 mutation toward fixation. This drift component of the fixation process is consistent with the 129 occasional appearance of vestigial pelvises during early lineage II samples when mean pelvic 130 score remained near 3 (Table S2, Figure 2). A delay of $\sim 3500$ years before pelvic reduction is 131 within the range of simulated lag times in pelvic reduction found by population genetic modeling 132 by Xie et al. ${ }^{12}$ (see figure 4D in ${ }^{12}$ and figures S6 and S7 in the Supplementary Materials of ${ }^{12}$ ), 133 given known mutation rates in the $\mathrm{Pel}$ enhancer region, reasonable selection coefficients for 134 pelvic loss $(0.1>\mathrm{s}>0.01)$, and relevant G. aculeatus population sizes. They found that the 135 probability of generating and fixing a de novo mutation in a fragile genome region was 1.0 for 136 reasonable stickleback population sizes $\left(10^{3}<\mathrm{N}<10^{6}\right)$ within 10,000 generations. When 137 selection was $\mathrm{s}=0.01$, predicted time to fixation was less than 5000 generations. When selection 138 was s $=0.1$, predicted time to fixation was less than 2000 generations. Assuming that $G$. 139 doryssus had a generation time of two years ${ }^{25}$, the observed delay of $\sim 3500$ years to begin 140 reduction and then another $\sim 3000$ years to reach a mode of PS 1 is reasonably close to 141 population genetic modeling for de novo mutation in the Pel enhancer region.

142 Thus, we conclude that major pelvic reduction in lineage II G. doryssus likely depended 143 on a new mutant (or very rare standing) recessive allele of Pitxl.

145 Question 2. Does immediate reduction in pelvic girdle length and pelvic spine length reveal the 146 presence of alleles of minor, additive effects? We used samples from a different temporal 147 sequence, $\mathrm{K}$ (Methods), but the same section of rock to answer this question. We first tested 148 whether pelvic girdle length and pelvic spine lengths declined immediately following the 149 replacement event of lineage I by lineage II (i.e., immediately have a negative slope for the trait 150 mean vs. time), while pelvic score delayed reduction (i.e., has an initial slope of zero). To do 151 this, we used a piecewise ("broken-stick") regression model, which estimates the slope and 152 intercept for two different pieces of a regression line, before and after a break point where the 153 slope is estimated to change significantly. Consistent with the visually obvious lag time before 154 pelvic score reduction (Figure 4), the first 'stick' inferred by the piecewise regression for pelvic 155 score against time had an intercept of 3.0 (the maximum possible pelvic score), a slope of zero, 
156 and a breakpoint between temporal samples five and six (Table 1). After this breakpoint, the 157 slope coefficient became negative (Table 1). In contrast, for fish with PS 3.0 (i.e., when the fully 158 functional dominant allele of Pitxl is hypothesized to be at high frequency), mean size-corrected 159 lengths of both pelvic girdle and pelvic spines began to decline immediately after appearance of 160 lineage II (Figure 4). The slope of the first 'stick' was significantly negative for both traits (Table 161 1). This implies available genetic variation unlinked to Pitxl.

162 Moreover, these significant trends for reduction of mean size-corrected pelvic girdle and 163 pelvic spine lengths both resulted from gradual shifts to smaller sizes by unimodal (Figure 5, 164 Table S3), normally distributed frequency distributions (Table S3). This finding is consistent 165 with multiple genes acting additively. Pearson correlations between pelvic girdle and pelvic 166 spine lengths calculated for each of the first ten samples in temporal sequence $\mathrm{K}$ averaged only $1670.38(\mathrm{sd}=0.28 ; \max =0.74 ; \min =-0.19)$, suggesting that the two traits might be reduced in part 168 via different genetic changes. A QTL study in G. aculeatus from a cross between populations 169 with complete and missing pelvises found that the two traits shared four QTL for length, but that 170 pelvic spine length also has a unique QTL that explains 5.6\% of its variance ${ }^{9}$. Thus, in that QTL 171 cross at least, there was potential for independent variation in the lengths of pelvic spine and 172 pelvic girdle, consistent with observation in the fossils.

173 We further measured pelvic vestige lengths for a subsample of 305 fossils with PS 1.0

174 (Table S4) - that is, individuals likely to have been homozygous for a null allele of Pitxl in the 175 pelvis. The distribution of lengths did not deviate from unimodal (Dip statistic $D_{n}=0.02, \mathrm{p}=$ 176 0.67). Last, we note that the pelvis did not completely disappear once the hypothesized deletion 177 mutations arose in Pitxl; i.e., PS 0 was not common. The persistence of intermediate pelvic 178 scores (i.e., PS 2.8 to 1.2; Figure 2, Figure 3) and the unimodal distribution of vestigial pelvic 179 girdle lengths in fish with PS $=1.0$ further suggest that other genes besides Pitx 1 were also 180 involved in pelvic development and reduction in G. doryssus.

181 Thus, we infer that Pitxl likely was not the sole genetic cause for pelvic reduction in 182 lineage II. Pelvic reduction also involved a suite of additive alleles with small effects. Such 183 alleles in lineage II G. doryssus would rarely produce strong pelvic reduction in any one 184 individual and could be carried even when selection favored full pelvises, as in the putative 185 source population of lineage $\mathrm{II}^{2}$. However, once selection for pelvic reduction began in lineage II 186 of G. doryssus (i.e., following appearance of lineage II to our depositional environment), these 
187 loci would have facilitated immediate reduction of pelvic girdle and pelvic spine lengths (Figure 188 4).

189 This inference is consistent with evolution in G. aculeatus, in which quantitative trait loci

190 (QTL) with small, additive effects on pelvic elements contribute to pelvic reduction ${ }^{9-11,18}$.

191 However, we note that there are phenotypic differences in the order of structural reduction of the 192 pelvic skeleton between extant G. aculeatus and fossil G. doryssus ${ }^{26}$. In G. doryssus, the pelvic

193 spine is lost first, at which point the pelvic girdle breaks into separate anterior and posterior

194 elements that correspond to different developmental structures ${ }^{27}$. The size of the vestigial

195 posterior element can vary in the fossils, but it is usually absent. In fossil specimens with PS 1.0

196 (i.e., no posterior element), the anterior element varies in size and can also be lost unilaterally or

197 on both sides. In contrast, in extant G. aculeatus, pelvic reduction usually proceeds through loss

198 of the pelvic spine without the pelvic girdle dividing into separate anterior and posterior

199 elements. Following spine loss, the posterior process gets shorter, leaving only a diminutive

200 ascending branch emanating from the anterior process. Next, the ascending branch gets shorter,

201 until it eventually leaves a structure that is indistinguishable from the tear-drop shaped anterior

202 element in the fossils. Finally, like the fossils, the anterior element is reduced in size and lost

203 unilaterally or bilaterally. These phenotypic differences in the order of loss and the separation of

204 anterior and posterior pelvic elements suggests that the number, identity, and expression of small

205 effect genes differs between G. aculeatus and G. doryssus. However, in both species, it is the

206 posterior half of the pelvic girdle that is most often reduced or missing. In G. aculeatus, the 207 posterior process develops separately from the anterior process ${ }^{27}$ and it is thus likely that the 208 posterior and anterior processes in G. doryssus also are underlain by separate developmental 209 modules.

\section{Discussion}

212 Despite being separated by 10 million years, our data suggest that G. aculeatus and G. doryssus

213 have both used Pitxl during evolution of major reduction of their pelvic armor. Inference of the

214 gene(s) responsible for skeletal change in the fossil record is very rare. For example, Schmid and

215 Villagra $^{28}$ attributed discontinuities in scale and skeletal variation among species of Triasssic

216 Saurichthys to two growth factors (i.e., Ectodysplasin, Fibroblast Growth Factor) or their

217 receptors. They argued that involvement of these genes in development of homologous structures 
218 in extant species implicates them in evolution of Saurichthys morphology. However, the

219 temporal, phylogenetic, and morphological differences between Saurichthys and the modern 220 analogues allows only preliminary conclusions. Similarly, Meredith et al. ${ }^{29}$ document repeated

221 transitions in the gene enamelin from a functional gene in extant mammals with enameled teeth

222 to a pseudogene in those lacking enamel or without teeth. They suggested enamelin was likely to

223 have been responsible for losses in the fossil record. Qu et al. ${ }^{30}$ made a similar argument for the

224 role of enamalin and several other genes during tooth gain and loss in stem osteichthyans.

225 Finally, Zhu and colleagues ${ }^{31,32}$ proposed that loss of sparcl in stem Chondrichthyans caused a 226 secondary loss of perichondral bone in that clade. We were not able to find additional, relevant 227 examples during a literature search in March 2019, searching "fossil gene*" and related queries 228 on scholar.google.com. (We did find, however, that that inferences of broader genetic 229 architecture responsible for change in the fossil record are more common $\left(e . g .{ }^{33-37}\right)$.)

230 This paucity of examples arises in part because claiming that a specific gene caused

231 phenotypic variation in a fossil lineage assumes that no other regions of the genome can generate

232 similar phenotypic effects. In other words, a plausible but ultimately untestable alternative

233 hypothesis exists: a different gene(s) was involved. Indeed, for stickleback, evidence is

234 accumulating that phenotypic parallelism does not necessarily imply genetic parallelism ${ }^{38,39}$.

235 However, for the following four reasons, we argue that our evidence meets a reasonable burden 236 of proof to infer the role of a specific gene, Pitx 1, in pelvic reduction in a fossil species. (i) First, 237 many genes involved in pelvis development also play a role in development elsewhere in the 238 body. Pitxl is no exception and is expressed in the jaw, pituitary gland, and other tissues during 239 development; mice with null mutations in the coding region of Pitx 1 die before birth or as 240 neonates and exhibit developmental abnormalities of the jaw, pituitary, and other structures ${ }^{7,40,41}$.

241 However, Pitxl stands out among candidates because its expression can be modulated 242 specifically in the pelvis without disrupting development elsewhere. Mutations to the PelA 243 enhancer region reduce expression of Pitx 1 in the pelvis ${ }^{7,12}$. (ii) Second, reduction of Pitx 1 244 expression is clearly involved in generating left-larger asymmetry in hindlimb elements through 245 Pitxl's interaction with Pitx $2^{15}$. Pitx2 is one of only six genes known to generate left-larger 246 directional asymmetry in vertebrate lateral plate mesoderm, the source of limb buds ${ }^{14}$. (iii) Third, 247 the PelA enhancer of Pitx 1 lies in a fragile portion of the genome that shows mutation rates $\sim 4$ 248 orders of magnitude higher than background ${ }^{12}$ and shows signatures of positive natural 
249 selection ${ }^{7,12}$, suggesting that favored variation might arise often at this locus. (iv) Fourth, pelvic 250 loss in Canadian and European populations of ninespine stickleback (Pungitius pungitius) maps

251 to Pitxl, suggesting that a parallel genetic mechanism for pelvic loss persisted across at least 7.2 252 to 6.9 million years of divergence from a common ancestor with G. aculeatus ${ }^{42,43}$; this timescale 253 is similar to our comparison between fossil G. doryssus and G. aculeatus. This result, combined 254 with the repeated use of Pitx 1 during pelvic loss by multiple independent populations of $G$. 255 aculeatus as well as in manatees ${ }^{9,11,16}$ suggests that Pitx 1 's role in pelvic reduction can be 256 remarkably parallel across distantly related and phenotypically diverse vertebrates. Thus, though 257 we can never disprove the alternative hypothesis that a different gene causes parallel phenotypic 258 outcomes in fossil G. doryssus and extant G. aculeatus, we feel that such a hypothesis is less 259 plausible than the simpler conclusion: Pitxl is the likely gene of major effect in this fossil 260 system.

262 Methods

263 The Fossil System

264 The fossil stickleback Gasterosteus doryssus (Extended Data 1) is abundant and well preserved 265 in a Miocene (10 million year old) lake deposit with annual layers, providing both excellent 266 samples and fine temporal resolution (reviewed by ${ }^{2}$ ). We focused on the evolution of lineage II

267 because we could observe evolution from an armored form, with full pelvic girdles and both 268 pelvic spines, to a vestigial form with reduced pelvic girdles and fewer, smaller pelvic spines.

270 Location and fossil sampling

271 Fossil G. doryssus were collected from an open pit, diatomaceous earth mine at $39.526^{\circ} \mathrm{N}$, $272119.094^{\circ} \mathrm{W}$, near Reno, Nevada, USA. In the field, we used sharpened putty knives to split the 273 rock along arbitrary bedding planes to find fossils. Each fossil's approximate stratigraphic 274 position was measured in relation to volcanic ash layers. Specimens were prepared in the 275 laboratory under a dissecting microscope, using probes to remove the matrix that covered 276 bones ${ }^{1,3}$. All specimens of G. doryssus, as well as lithological samples and associated field notes, 277 have been deposited in University of California Museum of Paleontology. 
280 Note that a 'section' is a span of stratigraphic thickness of rock. A 'sample' comprises multiple 281 fossil specimens that are all mined from the same section. Multiple samples make up a 'temporal 282 sequence'.

283 Fossil stickleback specimens used in this study come from two temporal sequences, $\mathrm{K}^{2}$

284 and $\mathrm{L}^{3}$, which comprise separate specimens collected with different sampling designs. However,

$285 \mathrm{~K}$ and $\mathrm{L}$ came from the same stratigraphic section in the same exposure, they overlap in time, 286 and they occupy the upper $17 \%$ of the stratigraphic section covering temporal sequence $\mathrm{D}$, 287 reported by ${ }^{1}$. D includes 26 samples made mostly at 5000 -year intervals and spans an estimated 288 108,275 years (Extended Data 2). Temporal sequence K spans 16,363 years (Extended Data 2) 289 and comprises 18 samples made at about 1000 year intervals (Table S4, Table S5). Each sample 290 was made from a narrow time interval of one to several consecutive years. $\mathrm{L}$ is one continuous 291 sequence spanning about 21,250 years (Extended Data 2). Following Bell et al. ${ }^{3}$, we binned 292 specimens from L into 250-year samples for analysis (Table S2). D, L, and K can be correlated 293 ( \pm 75 years) by aligning replacement of lineage I by lineage II observed in all three sequences.

294 This replacement event occurs $\sim 92,012$ years after the start of $D^{1}$ (Extended Data 2).

\section{Data use}

297 We used existing pelvic score data ${ }^{3}$ and new left-right pelvic vestige length data from lineage II 298 fossils from temporal sequence $\mathrm{L}$ to characterize the presence of pelvic-score multimodality and 299 directional asymmetry of pelvic vestiges (Question 1). We used existing and new data from 300 lineage II fossils from temporal sequence $\mathrm{K}$ to test whether the lengths of the pelvic girdle and 301 the pelvic spines began to decline immediately after lineage II replaced lineage I, and to infer 302 whether the evolution of these traits is consistent with polygenic, additive genetic architecture 303 (Question 2).

\section{Phenotyping}

306 Ordinal pelvic scores (PS) were assigned by MAB to pelvic phenotypes by visual inspection of 307 all fossils in both $\mathrm{L}$ and $\mathrm{K}$, using marked figures from reference ${ }^{26}$ as a standard (Extended Data 308 3). An individual with a full pelvic girdle (i.e., anterior and posterior processes, ascending 309 branch) and both pelvic spines present was scored PS 3.0. Reduction from 3.0 always starts with 310 loss of pelvic spines and concurrent division of the pelvic girdle into anterior and posterior 
311 elements on at least one side. Pelvic scores from 2.8 to 1.2 were assigned in intervals of 0.2

312 points based on the size and complexity of the posterior process of the pelvic girdle. Reduction

313 of PS from 1.2 to 1.0 indicates that the anterior pelvic plate vestige is present on at least one side

314 but posterior vestiges have been completely lost. The jump from PS 1.0 to PS 0 indicates loss of

315 anterior pelvic plate elements on both sides ${ }^{3}$. Extended Data 3 provides drawings from reference

$316{ }^{26}$ and photograph examples to illustrate how PS was scored. PS is significantly correlated with

317 digitized pelvic girdle area between PS 1.0 to $3.0\left(\mathrm{r}^{2}=0.82\right)^{3}$. Between PS 2.8 and 1.0, PS

318 mostly reflects a continuous distribution of size of the posterior process of the pelvic vestige. PS

319 compresses the phenotypic scale between scores of 0 and 1.0 because it does not take into

320 account whether one or both sides of the anterior pelvic vestige are present or the size of the

321 vestige within this range.

322 For specimens in temporal sequence $\mathrm{K}$, standard length was measured as the distance

323 from the tip of the upper jaw to the end of the last vertebra (hypural plate), using 'measure mode'

324 in tpsDIG ${ }^{44}$ on digital images of each fossil. Specimens with gaps between the vertebrae were

325 excluded, and protrusion of the premaxilla was taken into account. Standard length was often

326 measured in segments to limit the effect of postmortem (i.e., taphonomic) curvature of the

327 vertebral column. Pelvic girdle and pelvic spine lengths were also measured using tpsDIG.

328 Pelvic girdle lengths were measured differently depending on PS. Specimens with a full pelvis

329 (PS 3.0) were measured along the midline from the most anterior point of the anterior process to

330 the posterior tip of the posterior process of the pelvis on the side with the best preservation ${ }^{45}$. The

331 pelvic vestige of specimens with PS 1.0 was measured from the pointed anterior tip to the most

332 distal point on the rounded posterior edge. In specimens with PS 1.2 to 2.8, the length of the

333 posterior element along the median edge was measured and added to the length of the anterior

334 element. Specimens with no pelvic vestige (PS 0) were assigned a value of 0.0. Pelvic spine

335 lengths were measured from distal tip to the proximal base of the condyles by which they

336 articulate with the pelvic girdle.

337 For a subset of specimens in temporal sequence $\mathrm{L}$, the lengths of the anterior and

338 posterior pelvic vestiges were measured as described above for $\mathrm{K}$ specimens. However, unlike

339 for K, we measured both the right and left sides for specimens in which overlap of the vestiges

340 between sides allowed us to distinguish right from left. 


\section{Analysis}

343 All analysis was conducted in R version 3.6.1, (2019) ${ }^{46}$. Unless noted otherwise, statistical

344 functions come from this version's base 'stats' package. Functions are indicated by italics. The 345 statistical analysis described here was not preregistered.

347 Question 1. Is Pitx1 responsible for the major pelvic reduction observed in lineage II of G.

348 doryssus? We estimated directional asymmetry from the pelvic vestiges of temporal sequence L 349 specimens for which pelvic vestige lengths were measured on right and left sides. We excluded 350 fish with pelvic scores of 3.0, as they would not have had the hypothesized deletion mutation in 351 Pel that reduces Pitx 1 expression ${ }^{7,12}$ and analyzed only fish with pelvic scores less than 3.0 and 352 greater than or equal to 1.0. We summed the lengths of vestigial anterior and posterior pelvic 353 elements on the same side before quantifying length asymmetry between sides. For fish with 354 pelvic scores of 1 (i.e., no posterior elements), we compared length asymmetry in anterior 355 elements only.

Directional asymmetry was calculated as percent asymmetry,

$$
[\mathrm{rpv}-\mathrm{lpv}] /[\mathrm{rpv}+\mathrm{lpv}] * 100,
$$

where rpv and lpv are the right and left pelvic vestige lengths, respectively. Thus, specimens with a larger left vestige had negative asymmetry values. We used a two-sided paired t-test (t.test) to test whether right versus left pelvic vestige lengths are significantly asymmetric. We used a two-sided Chi-square test (chisq.test) to test whether the number of specimens with larger 362 right or left vestiges deviated significantly from $50 \%$.

363 We also used a two-sided Chi-square to test whether the frequencies of specimens with 364 larger and smaller left vestiges were influenced by pelvic score. That is, we asked if frequency 365 distributions of vestiges with a larger pelvic vestige on the left or right side within each pelvic 366 score class (i.e., 1.0, 1.2, .. 2.6, 2.8) differed from the pooled frequency distribution (Table S1). 367 If all vestigial pelvic phenotypes (i.e., PS 1.0 to 2.8) are caused by reduction of Pitx 1 expression 368 during pelvic girdle development, we would not expect the frequency distribution to vary among 369 pelvic score categories 9 .

370 Finally, if recessive alleles of a gene of large effect (i.e., Pitxl) underlie pelvic score 371 evolution, then we would expect frequency distributions of pelvic score to have discrete peaks, 372 deviating from unimodality. We verbally described the reduction in mean pelvic score through 
373 time using the fine-scale temporal resolution of temporal sequence L. We illustrated the change

374 in frequency distributions of pelvic score through time within temporal sequence K, using its

375 chronologically discrete and more sparse sampling for clearer plotting and presentation (Figure

$3763)$.

378 Question 2. Does reduction in continuous pelvic traits implicate the action of genes with minor, 379 additive effects?

380 To examine the role of minor genes in $G$. doryssus pelvic evolution, we measured the 381 lengths of one pelvic spine and of the pelvis (as described above) in temporal sequence $\mathrm{K}$ 382 specimens with PS 3.0. We used just PS 3.0 individuals to infer whether genes with small effects 383 contributed to pelvic reduction before a gene with major effects on PS arose to obscure the 384 effects of the minor genes. We restricted our analysis to the first 10 samples of $\mathrm{K}$, as only those 385 samples included enough specimens (i.e., 5 or more) with full pelvic scores to compute 386 reasonable means for pelvic spine and pelvic girdle lengths. Lengths for both continuous traits were size-corrected using standard length, following ${ }^{47-49}$ (Supplementary Information).

388 We plotted means for pelvic score and the two size-corrected traits against time to 389 visualize the timing of reduction of pelvic spine length, pelvic girdle length, and pelvic score 390 after lineage II appeared. For statistical support, we fit piecewise regressions (i.e., "broken stick" 391 models) to the trait means. If pelvic girdle and spine lengths dropped immediately while pelvic 392 score remained static, the first 'stick' for both pelvic spine and girdle lengths would have a 393 significantly negative slope, while the first stick for pelvic score would have a slope of zero. For 394 each trait, we modelled the linear relationship between mean trait values and time since lineage 395 II first appeared. Each model allowed one breakpoint along the temporal sequence of samples, 396 such that we estimated two sets of slopes and intercepts before and after the proposed breakpoint. 397 For each trait, we iterated through models that differed by where in the temporal sequence the 398 breakpoint was proposed. Then we chose the model with the lowest residual error as our best 399 estimate for the first temporal breakpoint. We limited our potential breakpoints to the first seven 400 samples because visual inspection suggests that significant differences in 'first stick' slope 401 between pelvic score and the other traits occur in this span (Figure 4). Moreover, the eighth 402 sample contains an increase in trait means (Figure 4). We note that with only seven values (i.e., 
seven samples), significance tests of slope and intercept have low power. Thus, we are mainly interested in the sign and estimate of the model parameters.

Next we plotted the frequency distributions for pelvic spine length and pelvic girdle length within each of the first 10 temporal samples for only individuals with PS 3.0. If the minor alleles underlying evolution of these traits are additive, we would expect these distributions to be normal and unimodal. We used Shapiro-Wilk Normality tests (shapiro.test) within each sample for each trait to test for deviations from normality. Complementarily, we used Hartigan's Dip

410 Statistic, $D_{n}{ }^{50}$, to test for deviations from unimodality (dip.test in the package 'diptest' ${ }^{51}$ ). We

411 calculated Pearson correlations (cor) to quantify the relationship between pelvic spine length and

412 pelvic girdle length for each sample; a strong correlation might imply the same genetic

413 mechanism for reduction. Finally, we pooled all individuals from temporal sequence K with PS

4141.0 (i.e., likely homozygous for the null Pitxl allele) and, as above, asked whether pelvic girdle

415 length was unimodal and normal. If so, it would further corroborate evidence that minor alleles

416 contributed to pelvic reduction.

Data and Code Availability

419 Data and code are available at datadryad.org (https://doi.org/10.5061/dryad.02v6wwq18 420 ).

\section{References}

422 1. Bell, M. A., Baumgartner, J. V. \& Olson, E. C. Patterns of temporal change in single morphological characters of a Miocene stickleback fish. Paleobiology 11, 258-271 (1985).

2. Bell, M. A. Implications of a fossil stickleback assemblage for Darwinian gradualism. Journal of Fish Biology 75, 1977-1999 (2009).

3. Bell, M. A., Travis, M. P. \& Blouw, D. M. Inferring natural selection in a fossil threespine stickleback. Paleobiology 32, 562-577 (2006).

4. Hunt, G., Bell, M. A. \& Travis, M. P. Evolution toward a new adaptive optimum: phenotypic evolution in a fossil stickleback lineage. Evolution 62, 700-710 (2008).

5. Klepaker, T., Østbye, K. \& Bell, M. A. Regressive evolution of the pelvic complex in stickleback fishes: a study of convergent evolution. Evolutionary Ecology Research 15, 413-435 (2013).

6. Bell, M. A., Ortí, G., Walker, J. A. \& Koenings, J. P. Evolution of pelvic reduction in threespine stickleback fish: a test of competing hypotheses. Evolution 47, 906-914 (1993).

7. Chan, Y. F. et al. Adaptive evolution of pelvic reduction in sticklebacks by recurrent deletion of a Pitxl enhancer. Science 327, 302-305 (2010). 
8. Cole, N. J., Tanaka, M., Prescott, A. \& Tickle, C. Expression of limb initiation genes and clues to the morphological diversification of threespine stickleback. Current Biology 13, R951-R952 (2003).

9. Shapiro, M. D. et al. Genetic and developmental basis of evolutionary pelvic reduction in threespine sticklebacks. Nature 428, 717 (2004).

10. Cresko, W. A. et al. Parallel genetic basis for repeated evolution of armor loss in Alaskan threespine stickleback populations. Proc. Natl. Acad. Sci. U.S.A. 101, 6050-6055 (2004).

11. Coyle, S. M., Huntingford, F. A. \& Peichel, C. L. Parallel evolution of Pitx 1 underlies pelvic reduction in Scottish Threespine Stickleback (Gasterosteus aculeatus). Journal of Heredity 98, 581-586 (2007).

12. Xie, K. T. et al. DNA fragility in the parallel evolution of pelvic reduction in stickleback fish. Science 363, 81-84 (2019).

13. Bell, M. A., Khalef, V. \& Travis, M. P. Directional asymmetry of pelvic vestiges in threespine stickleback. Journal of Experimental Zoology Part B: Molecular and Developmental Evolution 308B, 189-199 (2007).

14. Palmer, A. R. Symmetry breaking and the evolution of development. Science 306, 828833 (2004).

15. Marcil, A., Dumontier, É., Chamberland, M., Camper, S. A. \& Drouin, J. Pitx1 and Pitx2 are required for development of hindlimb buds. Development 130, 45-55 (2003).

16. Shapiro, M. D., Bell, M. A. \& Kingsley, D. M. Parallel genetic origins of pelvic reduction in vertebrates. Proc. Natl. Acad. Sci. U.S.A. 103, 13753-13758 (2006).

17. Bell, M. A. in The Evolutionary Biology of the Threespine Stickleback (eds. Bell, M. A. \& Foster, S. A.) 438-471 (Oxford University Press, 1994).

18. Peichel, C. L. et al. The genetic architecture of divergence between threespine stickleback species. Nature 414, 901-905 (2001).

19. Rollins, J. L., Lohman, B. K. \& Bell, M. A. Does ion limitation select for pelvic reduction in threespine stickleback (Gasterosteus aculeatus)? Evolutionary Ecology Research 16, 101-120 (2014).

20. Reimchen, T. E. Spine deficiency and polymorphism in a population of Gasterosteus aculeatus: an adaptation to predators? Canadian Journal of Zoology 58, 1232-1244 (1980).

21. Reimchen, T. E. in The Evolutionary Biology of the Threespine Stickleback (eds. Bell, M. A. \& Foster, S. A.) 240-276 (Oxford University Press, 1994).

22. Hoogland, R., Morris, D. \& Tinbergen, N. The spines of sticklebacks (Gasterosteus and Pygosteus) as a means of defense against predators (Perca and Esox). Behaviour 10, 205236 (1956).

23. Baumgartner, J. V. A new fossil ictalurid catfish from the Miocene middle member of the Truckee Formation, Nevada. Copeia 1982, 38-46 (1982).

24. Stearley, R. F. \& Smith, G. R. Fishes of the Mio-Pliocene western Snake River plain and vicinity. Miscellaneous Publications Museum of Zoology of the University of Michigan 204, 1-43 (2016).

25. Baker, J. A. in The Evolutionary Biology of the Threespine Stickleback (eds. Bell, M. A. \& Foster, S. A.) 144-187 (Oxford University Press, 1994).

26. Bell, M. A. Interacting evolutionary constraints in pelvic reduction of threespine sticklebacks, Gasterosteus aculeatus (Pisces, Gasterosteidae). Biological Journal of the Linnean Society 31, 347-382 (1987). 
27. Bell, M. A. \& Harris, E. I. Developmental osteology of the pelvic complex of Gasterosteus aculeatus. Copeia 1985, 789 (1985).

28. Schmid, L. \& Villagra, M. R. S. Potential genetic bases of morphological evolution in the triassic fish Saurichthys. Journal of Experimental Zoology Part B: Molecular and Developmental Evolution 314B, 519-526 (2010).

29. Meredith, R. W., Gatesy, J., Murphy, W. J., Ryder, O. A. \& Springer, M. S. Molecular decay of the tooth gene Enamelin (ENAM) mirrors the loss of enamel in the fossil record of placental mammals. PLoS Genetics 5, e1000634 (2009).

30. Qu, Q., Haitina, T., Zhu, M. \& Ahlberg, P. E. New genomic and fossil data illuminate the origin of enamel. Nature 526, 108-111 (2015).

31. Zhu, M. et al. A Silurian placoderm with osteichthyan-like marginal jaw bones. Nature 502, 188-193 (2013).

32. Zhu, M. Bone gain and loss: insights from genomes and fossils. National Science Review 1, 490-497 (2014).

33. Hunt, G. Evolutionary divergence in directions of high phenotypic variance in the Ostracode genus Poseidonamicus. Evolution 61, 1560-1576 (2007).

34. Thompson, J. R. et al. Reorganization of sea urchin gene regulatory networks at least 268 million years ago as revealed by oldest fossil cidaroid echinoid. Scientific Reports $\mathbf{5}$, 15541 (2015).

35. Organ, C. L., Janes, D. E., Meade, A. \& Pagel, M. Genotypic sex determination enabled adaptive radiations of extinct marine reptiles. Nature 461, 389-392 (2009).

36. Organ, C. L., Shedlock, A. M., Meade, A., Pagel, M. \& Edwards, S. V. Origin of avian genome size and structure in non-avian dinosaurs. Nature 446, 180-184 (2007).

37. Organ, C. L. \& Shedlock, A. M. Palaeogenomics of pterosaurs and the evolution of small genome size in flying vertebrates. Biology Letters 5, 47-50 (2009).

38. Conte, G. L., Arnegard, M. E., Peichel, C. L. \& Schluter, D. The probability of genetic parallelism and convergence in natural populations. Proc. Biol. Sci. 279, 5039-5047 (2012).

39. Rennison, D. J., Stuart, Y. E., Bolnick, D. I. \& Peichel, C. L. Ecological factors and morphological traits are associated with repeated genomic differentiation between lake and stream stickleback. Philos. Trans. R. Soc. Lond., B, Biol. Sci. 374, 20180241 (2019).

40. Szeto, D. P. et al. Role of the Bicoid-related homeodomain factor Pitx1 in specifying hindlimb morphogenesis and pituitary development. Genes Dev. 13, 484-494 (1999).

41. Thompson, A. C. et al. A novel enhancer near the Pitx1 gene influences development and evolution of pelvic appendages in vertebrates. eLIFE 7, e38555 (2018).

42. Bell, M. A., Stewart, J. D. \& Park, P. J. The world's oldest fossil Threespine Stickleback fish. Copeia 2009, 256-265 (2009).

43. Rawlinson, S. E. \& Bell, M. A. A stickleback fish (Pungitius) from the Neogene Sterling Formation, Kenai Peninsula, Alaska. Journal of Paleontology 56, 583-588 (1982).

44. Rohlf, F. J. tpsDIG ver. 2.10. (2006).

45. Bowne, P. S. in The Evolutionary Biology of the Threespine Stickleback (eds. Bell, M. A. \& Foster, S. A.) 28-60 (Oxford University Press, 1994).

46. R Core Team. R: A language and environment for statistical computing.

47. Lleonart, J., Salat, J. \& Torres, G. J. Removing allometric effects of body size in Morphological analysis. Journal of Theoretical Biology 205, 85-93 (2000). 
48. Oke, K. B. et al. Does plasticity enhance or dampen phenotypic parallelism? A test with three lake-stream stickleback pairs. J. Evol. Biol. 29, 126-143 (2016).

49. Stuart, Y. E. et al. Contrasting effects of environment and genetics generate a continuum of parallel evolution. Nature Ecology and Evolution 1, 0158 (2017).

50. Hartigan, J. A. \& Hartigan, P. M. The Dip Test of Unimodality. The Annals of Statistics 13, 70-84 (1985).

51. Maechler, M. diptest: Hartigan's dip statistic for unimodality - corrected. version 0.75-7 (2016).

Acknowledgements: Cyprus Industrial Minerals, CR Minerals, and T. Sumner of World Minerals permitted us to collect fossils on their property. We thank the people acknowledged in ${ }^{3}$ plus D. Arcieri, K. Brudvik, N.J. Buck, F. Castelli, R. Obeng, J. Qiao, C. Redman, and T.T. Zhang for field and laboratory assistance; F.J. Rohlf for statistical advice; M.D. Houseman for sharing his extensive knowledge of Truckee Formation paleoecology and taphonomy. C.W. Chan measured all pelvic vestiges for asymmetry analysis. We thank J. Jernvall, D.M. Kingsley, S. Chenoweth, R. Bonduriansky, Y.F. Chan, and S. Swank for helpful comments and discussion. This research was supported by NSF BSR-8111013, EAR-9870337, and DEB-0322818, Center for Field Research (Earthwatch), the National Geographic Society (2869-84), and NIH R01 GM124330-01 to M.A. Bell and by NSF DEB-1456462 and DEB-2003457 to Y.E. Stuart. This is contribution XXXX from Department of Biological Sciences, Rowan University.

Author contributions: MAB, MPT, and YES designed the research. MAB supervised sample and data collection. MPT collected the data. YES analyzed data, created figures, and wrote the paper. MAB, MPT, and YES jointly edited the paper. 


\section{Figures legends}

Figure 1. The pelvic vestige was larger on the left side in significantly more than half of all specimens of $G$. doryssus with vestigial pelvic structures. The magnitude of asymmetry was greater when the left vestige was larger than when the right vestige was larger. Asymmetry of pelvic vestiges was calculated for 877 specimens from temporal sequence L. Each vertical bar shows asymmetry for one specimen. The vertical line represents zero asymmetry. Individuals to the left of the line have larger left vestiges.

Figure 2. Mean pelvic score declines through time in temporal sequence L after a delay. The last sample in which every fish had a pelvic score of 3.0 occurred 2,500 years after lineage II replaced lineage I (Table S2). Reduction accelerated about 3,750 years after replacement (Table S2). Means minus one standard error are shown.

Figure 3. Relative frequency distributions of pelvic score through time from temporal sequence K. Pelvic score is multimodal, suggesting Mendelian expression of Pitx l during pelvic reduction. Analysis and discussion in the main text describe the more finely resolved sampling of temporal sequence L. Samples from K are plotted here for ease of visualization. The patterns are qualitatively the same. $\mathrm{k}$. T is the complete replacement of lineage I by lineage II. Time proceeds down the first column and then into the second column. Mean deposition time since the replacement event for each section is reported in years, as well as the mean pelvic score in the sample. Lines represent the proportion of specimens in each pelvic score category. Numbers above the lines are counts.

Figure 4. Reduction of size-adjusted pelvic girdle length (pgl) and pelvic spine length (psl) in temporal sequence $\mathrm{K}$ began immediately following replacement of lineage I by lineage II. In contrast, pelvic score, did not evolve substantially for another $\sim 3750$ years. Mean values are plotted with standard error bars. Arrows denote the first inferred breakpoint for each trait from piecewise regression. The slope of the first 'stick' is significantly negative for both pelvic girdle length and pelvic spine length. The slope of the first 'stick' for pelvic score is zero. Sample sizes are in Table S5.

Figure 5. Frequency distributions of (A) pelvic girdle length and (B) pelvic spine length for specimens with pelvic scores of 3.0 from temporal sequence K. Unimodality and normality suggest that multiple genes with additive effects underlie evolution in these traits. Time proceeds down. The oldest sample in this sequence is k.T, just after transition between lineage I and lineage II. Years since lineage II appeared are reported for each sample (Table S5), after which individuals with PS 3.0 become too rare to calculate a reasonable mean (Table S5). Black dots indicate sample means. 
Tables

599 Table 1. Piecewise regression models using data from temporal sequence K confirm that

600 reduction of pelvic girdle length and pelvic spine length began in lineage II immediately after it

601 replaced lineage I. In contrast, pelvic score had a lag time of $\sim 3750$ years before reduction began.

\begin{tabular}{lcccc}
\multicolumn{1}{c}{ (A) Pelvic girdle length } & $\begin{array}{c}\text { Coefficient } \\
\text { estimate }\end{array}$ & $\begin{array}{c}\text { Standard } \\
\text { error }\end{array}$ & t-value & P \\
\hline After-break intercept & 11.68 & 0.118 & 98.60 & $<0.001$ \\
Time $^{\dagger}$ & $-5.14 \times 10^{-4}$ & $3.3 \times 10^{-5}$ & -15.7 & 0.001 \\
Before-break* $^{*}$ & 1.09 & 0.163 & 6.7 & 0.006 \\
Time $\times$ Before-break time $\ddagger$ & $-1.34 \times 10^{-3}$ & $5.95 \times 10^{-4}$ & -2.3 & 0.109 \\
\hline
\end{tabular}

* Add to after-break intercept for before-break intercept: $11.68+1.09=12.77$

$\dagger$ The slope of the after-break 'stick': $-5.14 \times 10^{-4}$

+ Add to after-break slope $(\dagger)$ for before-break slope: $-5.14 \times 10^{-4}+-1.34 \times 10^{-3}=\mathbf{- 1 . 8 6} \times \mathbf{1 0}^{-3}$ Model significance: $F_{3,3}=326.2, P=0.0003$, Adj. $R^{2}=0.99$

\begin{tabular}{|c|c|c|c|c|}
\hline (B) Pelvic spine length & $\begin{array}{c}\text { Coefficient } \\
\text { estimate }\end{array}$ & $\begin{array}{c}\text { Standard } \\
\text { error }\end{array}$ & t-value & $\mathrm{P}$ \\
\hline After-break intercept & 5.60 & 0.165 & 34.0 & $<0.001$ \\
\hline Time $\dagger$ & $-1.86 \times 10^{-4}$ & $4.54 \times 10^{-5}$ & -4.1 & 0.026 \\
\hline Before break* & 2.33 & 0.227 & 10.3 & 0.002 \\
\hline Time x Before-break time & $-3.75 \times 10^{-3}$ & $8.27 \times 10^{-4}$ & -4.5 & 0.020 \\
\hline \multicolumn{5}{|c|}{$\begin{array}{l}\text { * Add to global Intercept for before-break intercept: } 5.60+2.33=7.93 \\
+ \text { The slope of the after-break 'stick': }-1.86 \times 10^{-4} \\
+ \text { Add to after-break slope }(\dagger) \text { for before-break slope: }-1.86 \times 10^{-4}+-3.75 \times 10^{-3}=\mathbf{- 3 . 9 4} \times \mathbf{1 0}^{-3} \\
\text { Model significance: } F_{3,2}=126.8, P=0.001, \text { Adj. } R^{2}=0.98\end{array}$} \\
\hline (C) Pelvic score & $\begin{array}{c}\text { Coefficient } \\
\text { estimate }\end{array}$ & $\begin{array}{c}\text { Standard } \\
\text { error }\end{array}$ & t-value & $\mathrm{P}$ \\
\hline After-break intercept & 4.67 & 0.00 & $8.8 \times 10^{15}$ & $<0.001$ \\
\hline Time $\dagger$ & $-4.53 \times 10^{-4}$ & 0.00 & $-4.3 \times 10^{14}$ & $<0.001$ \\
\hline Before break * & -1.67 & 0.00 & $-3.1 \times 10^{14}$ & $<0.001$ \\
\hline Time $\mathrm{x}$ Before-break time $t$ & $4.53 \times 10^{-4}$ & 0.00 & $3.9 \times 10^{14}$ & $<0.001$ \\
\hline \multicolumn{5}{|c|}{$\begin{array}{l}* \text { Add to global Intercept for before-break intercept: } 4.67+-1.67=3.00 \\
\dagger \text { The slope of the after-break 'stick': }-4.53 \times 10^{-4} \\
+ \text { Add to after-break slope }(\dagger) \text { for before-break slope: }-4.53 \times 10^{-4}+4.53 \times 10^{-4}=\mathbf{0 . 0 0} \\
\text { Model significance: } \mathrm{F}_{3,3}=13.53, \mathrm{P}<0.001, \text { Adj. } \mathrm{R}^{2}=1\end{array}$} \\
\hline
\end{tabular}




\section{Figures legends}

Figure 1. The pelvic vestige was larger on the left side in significantly more than half of all specimens of $G$. doryssus with vestigial pelvic structures. The magnitude of asymmetry was greater when the left vestige was larger than when the right vestige was larger. Asymmetry of pelvic vestiges was calculated for 877 specimens from temporal sequence L. Each vertical bar shows asymmetry for one specimen. The vertical line represents zero asymmetry. Individuals to the left of the line have larger left vestiges.

Figure 2. Mean pelvic score declines through time in temporal sequence $\mathrm{L}$ after a delay. The last sample in which every fish had a pelvic score of 3.0 occurred 2,500 years after lineage II replaced lineage I (Table S2). Reduction accelerated about 3,750 years after replacement (Table S2). Means minus one standard error are shown.

Figure 3. Relative frequency distributions of pelvic score through time from temporal sequence K. Pelvic score is multimodal, suggesting Mendelian expression of Pitxl during pelvic reduction. Analysis and discussion in the main text describe the more finely resolved sampling of temporal sequence L. Samples from K are plotted here for ease of visualization. The patterns are qualitatively the same. $\mathrm{k}$.T is the complete replacement of lineage I by lineage II. Time proceeds down the first column and then into the second column. Mean deposition time since the replacement event for each section is reported in years, as well as the mean pelvic score in the sample. Lines represent the proportion of specimens in each pelvic score category. Numbers above the lines are counts.

Figure 4. Reduction of size-adjusted pelvic girdle length (pgl) and pelvic spine length (psl) in temporal sequence $\mathrm{K}$ began immediately following replacement of lineage I by lineage II. In contrast, pelvic score, did not evolve substantially for another $\sim 3750$ years. Mean values are plotted with standard error bars. Arrows denote the first inferred breakpoint for each trait from piecewise regression. The slope of the first 'stick' is significantly negative for both pelvic girdle length and pelvic spine length. The slope of the first 'stick' for pelvic score is zero. Sample sizes are in Table S5.

Figure 5. Frequency distributions of (a) pelvic girdle length and (b) pelvic spine length for specimens with pelvic scores of 3.0 from temporal sequence K. Unimodality and normality suggest that multiple genes with additive effects underlie evolution in these traits. Time proceeds down. The oldest sample in this sequence is k.T, just after transition between lineage I and lineage II. Years since lineage II appeared are reported for each sample (Table S5), after which individuals with PS 3.0 become too rare to calculate a reasonable mean (Table S5). Black dots indicate sample means. 